\title{
Deep Vein Thrombosis /Pulmonary Embolism in a Patient with Retroperitoneal Fibrosis: A Case Report
}

\author{
Harsh Agrawal ${ }^{1 *}$, Christine I Bishop ${ }^{1}$, Soma Wali ${ }^{1}$ and Mary L Sealey ${ }^{2}$
}

${ }^{1}$ Department of Internal Medicine, University of California, Los Angeles-Olive View Program, David Geffen School of Medicine, Los Angeles, CA ${ }^{2}$ Department of Internal Medicine, Alleghany General Hospital, Temple School of Medicine, Pittsburgh, PA

\begin{abstract}
Introduction: Retroperitoneal Fibrosis (RPF) is a clinicopathological condition characterized by inflammatory fibrotic reaction around infrarenal aorta, iliac vessels and surrounding retroperitoneum with myriad presentations. This case report shows how a Deep Vein Thrombosis (DVT) and subsequent Pulmonary Embolism (PE) can be a potential complication of this disease. A potential temporal association was seen with chronic beta blocker use and retroperitoneal fibrosis.
\end{abstract}

Case presentation: A 62-year-old Caucasian male with history of hypertension on chronic beta-blocker therapy for 2 years (Metoprolol $50 \mathrm{mg}$ twice daily) presented with sub-acute left sided non-radiating lower back pain of 5-day duration. Computerized Tomography (CT) scan with contrast of the abdomen and pelvis revealed large segment of inflammatory stranding involving the periaortic retroperitoneum extending from the level of the kidneys upto the pelvis and incasing the left lliac veins. CT guided retroperitoneal core biopsy was done which was consistent with retroperitoneal fibrosis. Two months later, patient presented with complain of acute onset shortness of breath and increasing lower extremity edema, on the left side. Electrocardiogram was consistent with a finding of new onset Atrial fibrillation (A fib). Ultra Sono Gram (USG) Doppler study of lower extremities unveiled occlusive left sided DVT of the popliteal vein. A CT Angiography demonstrated segmental and sub-segmental pulmonary emboli of the right lower lobe with no evident pulmonary edema. He was treated with steroids, digoxin and warfarin. On follow up a repeat CT scan three months later of the abdomen and pelvis showed stable retroperitoneal mass with no further progression.

Conclusion: Our patient presented with lower extremity edema and imaging revealed extension of RPF to involve common iliac vessels. With beta-blockers as a possible inciting event, RPF causing venous stasis, iliac vein compression and thus DVT/PE is the most plausible explanation This case reports add to the medical literature how DVT/PE can be cause by an underlying disease entity not related to the usual causes and if not worked up patients may be labeled as having unprovoked events. Any relationship between beta blockers and RFP is questionable and has not been proven in any randomized trials, but should be thought of by the physician if such clinical situation is encountered.

\section{Introduction}

Retroperitoneal Fibrosis (RPF) is a clinico pathological condition characterized by inflammatory fibrotic reaction around infra renal aorta, iliac vessels and surrounding retroperitoneum with myriad presentations [1].

Major advancements have been made since then towards understanding the disease process, though lack of definite diagnostic criteria and treatment protocol still remains a challenge.

RPF has estimated incidence of 1-2 per 100,000 [2]. Given the rarity of the condition the diagnosis may often be missed if not included in differential diagnosis in appropriate clinical background. It is imperative to identify and treat RPF early on in its course in order to avoid potentially devastating complications including Deep Vein Thrombosis, Pulmonary Embolism, reno-vascular hypertension, ureteric obstruction, Chronic Kidney Disease (CKD) [3]. Here we describe a case of RPF in a patient on chronic beta blocker therapy who later presented with unilateral DVT and PE. Our objectives are to consider RPF as one of the potential complication of chronic betablocker therapy and to recognize DVT/PE as a potential complication of RPF.

\section{Case Presentation}

A 62-year-old Caucasian male with history of hypertension on chronic beta-blocker therapy for 2 years (Metoprolol $50 \mathrm{mg}$ twice daily) presented with sub-acute left sided non-radiating lower back pain of 5-day duration. He had no history of trauma, fever, abdominal surgeries or any recent episodes of illness. On presentation he was a febrile and normotensive. Physical examination was non-revealing for any significant finding apart from mild bilateral pedal edema. Initial lab work showed White blood cell count of $12000 / \mathrm{mcl}$, platelets $400,000 /$ $\mathrm{mcl}$, and hemoglobin $15.1 \mathrm{gm} / \mathrm{dl}$. Metabolic profile, liver function tests and renal function tests were in normal range. Amylase and lipase were $26 \mathrm{mg} / \mathrm{dl}$ and $18 \mathrm{mg} / \mathrm{dl}$, respectively. Sedimentation Rate (ESR) was 20. C-Reactive Protein (CRP) was normal. Lower extremity doppler was not performed at this visit as the edema was described by the patient as long standing, symmetric and possibly form venous stasis given his obese body habitus. Blood and urine cultures were negative. Echocardiography revealed moderate LV dysfunction, with ejection fraction of $45 \%$. Computerized Tomography (CT) scan with contrast of the abdomen and pelvis revealed large segment of inflammatory stranding involving the periaortic retroperitoneum extending from the level of the kidneys upto the pelvis and incasing the left Iliac veins (Figure 1). Differential

*Corresponding author: Harsh Agrawal, Department of Internal Medicine University of California, Los Angeles-Olive View Program, David Geffen School of Medicine, Los Angeles, 14445 Olive View Drive, 2B-182, Sylmar CA, 91342, CA, USA, E-mail: harshagrawal@hotmail.com

Received May 31, 2012; Accepted June 13, 2012; Published June 23, 2012

Citation: Agrawal H, Bishop Cl, Wali S, Sealey ML (2012) Deep Vein Thrombosis /Pulmonary Embolism in a Patient with Retroperitoneal Fibrosis: A Case Report. J Clin Case Rep 2:154. doi:10.4172/2165-7920.1000154

Copyright: ( 2012 Agrawal $\mathrm{H}$, et al. This is an open-access article distributed under the terms of the Creative Commons Attribution License, which permits unrestricted use, distribution, and reproduction in any medium, provided the original author and source are credited. 
diagnosis included mesenteric infarct and lymphoma, and metastatic mass. Magnetic Resonance Imaging (MRI) showed abnormal soft tissue mass in left para-aortic region without any particular signal characteristics to help differentiation of the pathology. CT guided retroperitoneal core biopsy was done, which showed fibrofatty tissue admixed with lymphoplasmacytic infiltrate with scattered lymphoid aggregates accompanied by infiltration of eosinophils and numerous foamy macrophages. Immunohistochemistry demonstrated normal distribution of CD20 -positive B-cells and CD3- positive B-cells without coexpression of CD5. A diagnosis of RPF was made given the clinical, imaging and pathological presentation. Patient was started on prednisone $40 \mathrm{mg}$ daily. His beta- blocker therapy was stopped.

Two months later, patient presented with complain of acute onset shortness of breath and increasing lower extremity edema, on the left side. There was no history of trauma, long travel, infection or any other immediate precipitating event. Electrocardiogram was consistent with a finding of new onset Atrial fibrillation (A fib), which should be considered a red flag for possible new onset PE. Ultra Sono Gram (USG) Doppler study of lower extremities unveiled occlusive left sided DVT of the popliteal vein. A CT angiography demonstrated segmental and sub-segmental pulmonary emboli of the right lower lobe with no evident pulmonary edema (Figure 2). Coagulation panel was within normal range. Patient was treated accordingly for PE/DVT with heparin which was eventually bridged to warfarin and A fib with digoxin. His condition improved and he was discharged after 5 days. Patient is being followed up and a repeat CT scan three months later of the Abdomen and pelvis showed stable retroperitoneal mass with no further progression.

\section{Discussion}

RPF most commonly presents with dull, aching low back or flank pain. Systemic features of inflammation like weight loss, anorexia, fever, easy fatigability are common presenting symptoms. Patients can also present with complications secondary to local effects like new onset reno-vascular hypertension, lower extremity edema, hydrocoele, DVT [4]. Idiopathic cases account for nearly two third of the presentations.

Beta-blockers, including metoprolol and eye drops containing timolol have been reported to associate with RPF [5] others include Ergot derivatives [6], hydralazine [7], bromocriptine [8] and methyldopa

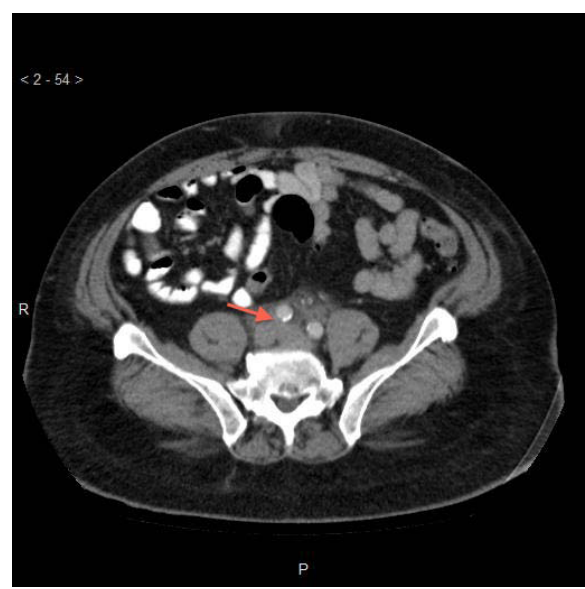

Figure 1: Computerized Tomographic (CT) scan showing the iliac veins incased by fibrosis (Red Arrow).

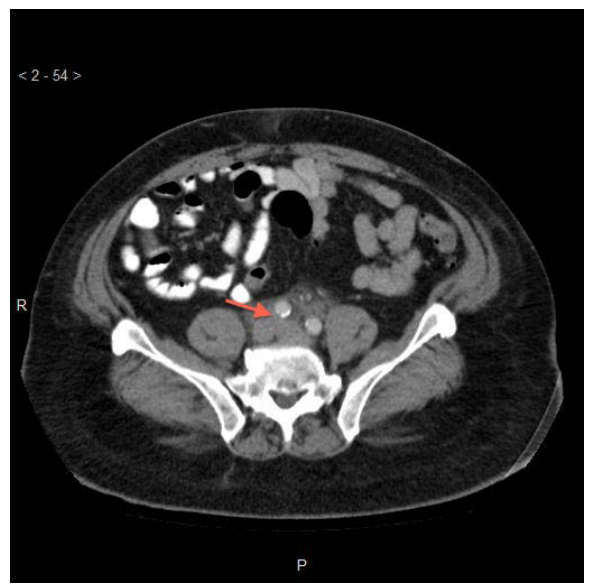

Figure 2: Computerized Angiography Revealing Sub-Segmental Pulmonary Arterial Emboli (Black Arrow).

[9], pergolide [10]. Certain malignancies like lymphomas, gastric and pancreatic cancers have been found to be associated with RPF [11]. Radiation therapy [3] has been found to be a significant risk factor associated with RPF. Certain infections like tuberculosis [12] actinomycosis [13] and schisostomiasis [14] have been reported as causative factors of RPF. Previous trauma in form of surgery has also been reported [15].

Although the etiopathogenesis is poorly understood, recent research studies points to the role of autoimmune mechanisms. Hypothesis proposed include excessive immune reaction in response to steroid and oxidized LDL molecules in local aortic atherosclerotic process [16]. Role of IgG4 bearing plasma cell is also proposed [17]. RPF has been described in association certain autoimmune diseases like thyroiditis and association with $\mathrm{HLA}-\mathrm{DRB} 1^{*} 03$ allele has been described [1].

Investigators have proposed a staging system based on the extent of the disease on imaging. Stage I RPF has fibrosis limited to infra-renal aorta or iliac vessels; stage II involves progression of fibrosis to inferior vena cava. Stage III further lateral extension of fibrosis occurs causing compression of the ureters and in stage IV the renal vessels are also involved. The same group of investigators has also proposed diagnostic criteria for idiopathic RPF with absence of any aneurismal dilatation of the aorta or absence of any mass in pelvic and abdominal cavity, in association with age specific cancer screening with normal outcomes [3].

Imaging remains the mainstay in diagnosing RPF. CT and MRI are most preferred modalities to identify and stage the disease process [18]. On MRI, RPF appears hypointense on T1 weighted images and hyperintense on $\mathrm{T} 2$ weighted images [1].

Fluorodeoxyglucose Positron Emission Technology (PET) is a non-invasive, safe, whole body imaging method with potential to identify other diseased sites, any associated autoimmune or neoplastic disorder and also can be used for surveillance of disease activity [19]. Biopsy can be done to identify histopathology of the disease [20]. It is generally required when infection or malignancy is strongly suspected, or location of lesion is atypical raising doubt about the diagnosis or surgical intervention is being planned.

Treatment of RPF can include both medical and surgical measures depending on the presentation. Stopping any possible inciting agent is the first step. Our patient had been on chronic beta-blocker 
therapy. It was stopped and in conjunction with steroid therapy disease process was stable on follow up after three months. Steroids are first line therapy if there is no evidence of any mass effect such as compression of surrounding structure, vascular extension into the surrounding veins and arteries, tubular compression like ureters or organ function compromise. Steroid resistant disease can be treated by other immunosuppressive agents including cyclophosphamide, methotrexate, mycophemolate mofetil [21] colchicine [22] and tamoxifen [23].

Follow up of disease activity can be done by serial imaging including contrast enhanced CT, MRI and PET. Measuring Serum Ig4 level [24] ESR/CRP [25] and renal function periodically may also be helpful, though the validity of these studies for following up of disease activity remains to be validated in larger studies. If the disease doesn't respond to these measures, diagnosis should be reconsidered and other causes must be ruled out. Complication like ureteral entrapment and consequent renal dysfunction is one of the most recognized complications of RPF [3]. Studies have shown that incidence of DVT and PE is also increased in patients with RPF and prophylactic anticoagulation should be considered on individual case by case basis in such patients with evidence of inferior vena cava and/or iliac vessel involvement causing venous stasis and lower extremity edema [3]. To our knowledge there have been no randomized trials evaluating the duration of treatment and prophylactic use of anticoagulation in this patient population. A strong argument can be made regarding more frequent surveillance i.e every 3 to 6 months, by different imaging modalities to access the progression of the disease instead of subjecting the patient to risks of anticoagulation including but not limited to hemorrhagic complications.

\section{Conclusion}

Our patient presented with lower extremity edema and imaging revealed extension of RPF to involve common iliac vessels. With betablockers as a possible inciting event, RPF causing venous stasis, iliac vein compression and thus DVT/PE is the most plausible explanation. A-fib is a very important symptom of PE especially in patients who had their beta blockers discontinued recently.

\section{References}

1. Vaglio A, Palmisano A, Corradi D, Salvarani C, Buzio C (2007) Retroperitoneal fibrosis: evolving concepts. Rheum Dis Clin North Am 33: 803-817, vi-vii.

2. Uibu T, Oksa P, Auvinen A, Honkanen E, Metsärinne K, et al. (2004) Asbestos exposure as a risk factor for retroperitoneal fibrosis. Lancet 363: 1422-1426.

3. Scheel PJ Jr, Feeley N (2009) Retroperitoneal fibrosis: the clinical, laboratory, and radiographic presentation. Medicine (Baltimore) 88: 202-207.

4. Vaglio A (2009) Retroperitoneal fibrosis: new insights into clinical presentation and diagnosis. Medicine (Baltimore) 88: 208-210.

5. Bullimore DW (1982) Retroperitoneal fibrosis associated with metoprolol. $\mathrm{Br}$ Med J (Clin Res Ed) 284: 664

6. (2002) Fibrosis due to ergot derivatives: exposure to risk should be weighed up. Prescrire Int 11: 186-189.

7. Waters VV (1989) Hydralazine, hydrochlorothiazide and ampicillin associated with retroperitoneal fibrosis: case report. J Urol 141: 936-937.

8. Herzog A, Minne H, Ziegler R (1989) Retroperitoneal fibrosis in a patient with macroprolactinoma treated with bromocriptine. BMJ 298: 1315.

9. Ahmad S (1983) Methyldopa and retroperitoneal fibrosis. Am Heart J 105: 1037-1038.

10. Apostolakis EE, Baikoussis NG, Tselikos D, Koniari I, Prokakis C, et al. (2009) Cardiac surgery in a patient with retroperitoneal fibrosis and heart valvulopathy, both due to pergolide medication for Parkinson's disease. J Cardiothorac Surg 4: 65 .

11. Vaglio A, Salvarani C, Buzio C (2006) Retroperitoneal fibrosis. Lancet 367 : 241-251.

12. Greco P, Vaglio A, Corradi D, Cobelli R, Zompatori M, et al. (2005) Tuberculosis as a trigger of retroperitoneal fibrosis. Clin Infect Dis 41: e72-75.

13. Milam MR, Schultenover SJ, Crispens M, Parker L (2004) Retroperitoneal fibrosis secondary to actinomycosis with no intrauterine device. Obstet Gynecol 104: 1134-1136.

14. Rollino C, Guzman H, Beltrame G, Ferro M, Quattrocchio G, et al. (2008) Retroperitoneal fibrosis and schistosomiasis: a causal relationship? Eur Intern Med 19: 297-299.

15. Katz R, Golijanin D, Pode D, Shapiro A (2002) Primary and postoperative retroperitoneal fibrosis-experience with 18 cases. Urology 60: 780-783.

16. Parums DV (1990) The spectrum of chronic periaortitis. Histopathology 16 423-431.

17. Oguchi T, Okada M, Endo F, Hattori K, Fujisaki A, et al. (2009) IgG4-related idiopathic retroperitoneal fibrosis: a case report. Hinyokika Kiyo 55: 745-748.

18. Vivas I, Nicolás AI, Velázquez P, Elduayen B, Fernández-Villa T, et al. (2000) Retroperitoneal fibrosis: typical and atypical manifestations. $\mathrm{Br} \mathrm{J}$ Radiol 73 : 214-222.

19. Piccoli GB, Consiglio V, Arena V, Pelosi E, Anastasios D, et al. (2010) Positron emission tomography as a tool for the 'tailored' management of retroperitoneal fibrosis: a nephro-urological experience. Nephrol Dial Transplant 25: 26032610 .

20. Cavalleri A, Brunner P, Monticelli I, Mourou MY, Bruneton JN (2008) CT-guided biopsy in two cases of retroperitoneal fibrosis. Clin Imaging 32: 230-232.

21. Swartz RD (2009) Idiopathic retroperitoneal fibrosis: a review of the pathogenesis and approaches to treatment. Am J Kidney Dis 54: 546-553.

22. Vega J, Goecke H, Tapia H, Labarca E, Santamarina M, et al. (2009) Treatment of idiopathic retroperitoneal fibrosis with colchicine and steroids: a case series. Am J Kidney Dis 53: 628-637.

23. Frankart L, Lorge F, Donckier J (1997) Tamoxifen for retroperitoneal fibrosis Postgrad Med J 73: 653-654.

24. Marumo K, Tatsuno S, Noto K (2009) Elevated serum IgG4 may predict sensitivity to steroid therapy in retroperitoneal fibrosis. Int J Urol 16: 427.

25. Magrey MN, Husni ME, Kushner I, Calabrese LH (2009) Do acute-phase reactants predict response to glucocorticoid therapy in retroperitoneal fibrosis? Arthritis Rheum 61: 674-679. 